Fermilab-0852-AD

\title{
Small-Amplitude Synchrotron Tune Near Transition
}

\author{
K.Y. Ng
}

May, 2010

\begin{abstract}
The separatrices of the rf buckets near transition are mapped when the synchronous phase is neither 0 or $\pi$. The small-amplitude synchronous tune is derived when the rf frequency is changed.
\end{abstract}




\section{Introduction}

Synchrotron radiation is present in all electron storage ring. As a result, the synchronous phase is always offset from $\phi_{s}=\pi$ to compensate for the power loss. Even for proton storage rings with negligible synchrotron radiation, the synchronous phase is also required to be offset from $\phi_{s}=0$ or $\pi$ slightly to compensate for beam loading. Thus for all storage rings operating near transition, beam particles reside in accelerating buckets instead of stationary bucket. It is of interest to map these buckets and see how they evolve near transition.

When the rf frequency is varied, the closed orbit is pushed radially inward or outward. The momentum of the particle synchronous with the rf is thus changed. By measuring the small-amplitude synchrotron tune as a function of the rf frequency, the lowest first few orders of the slip factor can be inferred. Here, we derive this relationship up to the lowest first three orders of the slip factor when the particle velocity is not ultra-relativistic.

\section{Equations of Motion}

The arrival time advance $\tau$ of a particle with fractional momentum spread $\delta=\left(p-p_{s}\right) / p_{s}$ is given by

$$
\frac{\tau}{T_{s}}=-\eta(\delta) \delta
$$

where the subscript 's' stands for on-momentum or synchronized with the rf. Thus $T_{s}$ is the revolution period of an on-momentum particle, and $\eta(\delta)$ is the slip factor, which can be expanded in $\delta$ as

$$
\eta(\delta)=\eta_{0}+\eta_{1} \delta+\eta_{2} \delta^{2}+\cdots
$$

The negative sign on the right-side of Eq. (2.1) is correct, because a particle with $\delta>0$ will arrive late above transition $(\eta>0)$. Let $T$ denote the revolution period of the test particle. Equation (2.1) can also be written as

$$
\frac{\Delta T}{T_{s}}=\frac{T-T_{s}}{T_{s}}=+\eta(\delta) \delta
$$

Note the positive sign on the right.

The energy equation is

$$
\Delta E=e V\left[\sin \phi-\sin \phi_{s}\right]
$$


where $V$ is the rf voltage, $\phi$ is the $\mathrm{rf}$ phase, while $\phi_{s}$ is the synchronous phase. Here $\Delta \phi=$ $\phi-\phi_{s}$ is the rf phase advance compared with the synchronous or on-momentum particle. This is the accumulation on the rf clock as the test particle reaches the rf cavity. Since the synchronous particle is behind by time $\tau$, we must have $\Delta \phi=-\omega_{\mathrm{rf}} \tau$. The negative sign comes about because a positive time advance $(\tau>0)$ implies the rf clock, that is synchronized with the synchronous particle by advancing the phase $2 \pi h$ with $h$ the rf harmonic, is lacking behind.

We first assume beam particles to be ultra relativistic, or $\beta \rightarrow 1$. Thus $\Delta E / E_{s} \rightarrow \delta$. After smoothing the discrete nature, the equations of motion become

$$
\begin{aligned}
\frac{d \Delta \phi}{d t} & =h \omega_{0 s}\left[\eta_{0}+\eta_{1} \delta+\cdots\right] \delta, \\
\frac{d \delta}{d t} & =\frac{e V}{E_{s} T_{s}}\left[\sin \left(\Delta \phi+\phi_{s}\right)-\sin \phi_{s}\right],
\end{aligned}
$$

where $\omega_{0 s}=2 \pi / T_{s}$ is the angular revolution of the synchronous or on-momentum particle. We next explore the situation when $\eta_{0}>0, \eta_{1}<0$, and neglect all higher-order slip factors. The fixed points are (see Fig. 1)

$$
\begin{aligned}
\delta_{f} & =0, \frac{\eta_{0}}{\left|\eta_{1}\right|}, \\
\Delta \phi_{f} & =2 n \pi,(2 n+1) \pi-2 \phi_{s} .
\end{aligned}
$$

Let us examine the fixed point $\left(\delta_{f}, \Delta \phi_{f}\right)=\left(0,2 n \pi-2 \phi_{s}\right)$. For small amplitude deviation, the equations of motion becomes

$$
\begin{aligned}
\frac{d \Delta \phi}{d t} & =h \omega_{0 s} \eta_{0} \delta, \\
\frac{d \delta}{d t} & =\frac{e V}{E_{s} T_{s}}\left[\sin \left(\Delta \phi+\phi_{s}\right)-\sin \phi_{s}\right]=\frac{e V \cos \phi_{s}}{E_{s} T_{s}} \Delta \phi .
\end{aligned}
$$

Eliminating $\Delta \phi$, one gets

$$
\frac{d^{2} \delta}{d t^{2}}=\frac{e h V \eta_{0} \cos \phi_{s}}{2 \pi E_{s}} \omega_{0 s}^{2} \delta
$$

Thus $(0,2 n \pi)$ is a stable fixed point if $\eta_{0} \cos \phi_{s}<0$, and the synchrotron tune is

$$
\nu_{s}=\sqrt{\frac{e h V \eta_{0} \cos \phi_{s}}{2 \pi E_{s}}}
$$

Obviously $\left(0,(2 n+1) \pi-2 \phi_{s}\right)$ is the unstable fixed point nearby. 


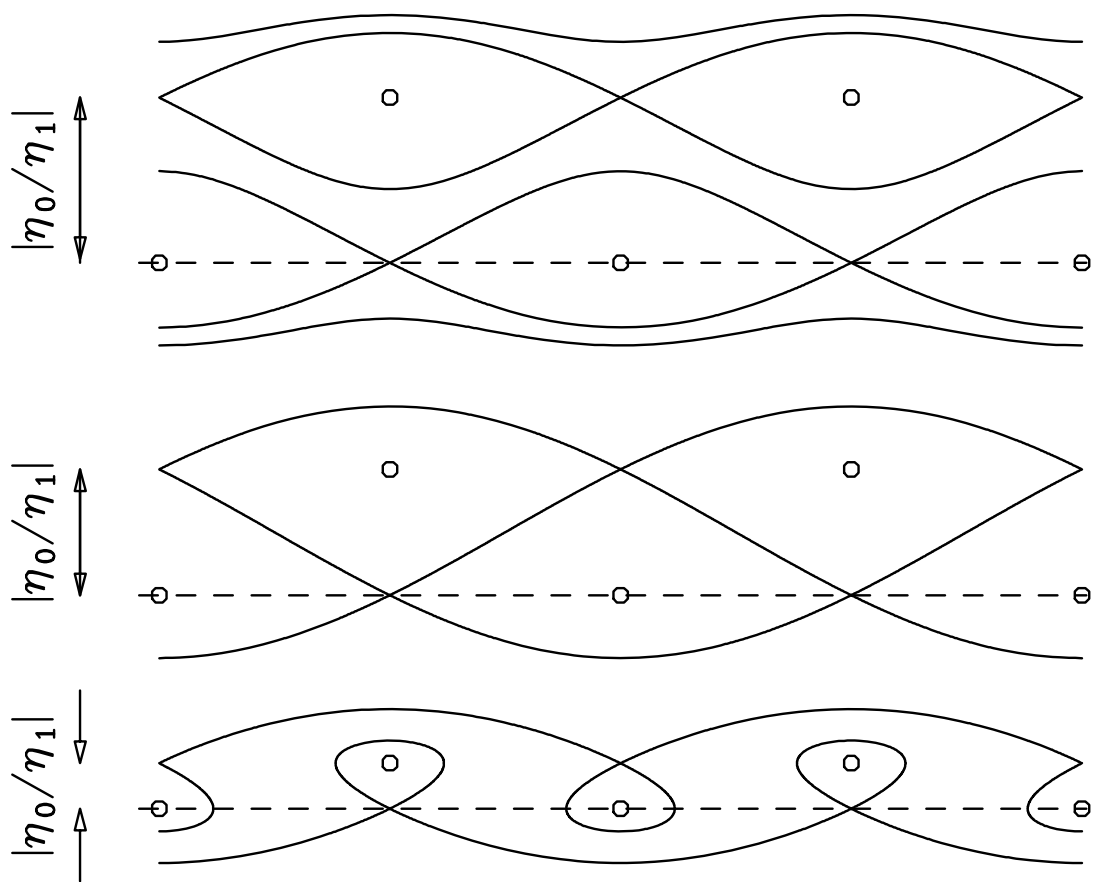

Figure 1: Plot of separatrices when $\phi_{s}=0$ or $\pi$ in the $\Delta \phi-\delta$ space. Top: When $\left|\eta_{0} / \eta_{1}\right|$ is larger than the critical value $\left|\frac{\eta_{0}}{\eta_{1}}\right|_{c}=\sqrt{\left|\frac{6 e V}{\pi \beta_{0}^{2} h \eta_{0} E_{s}}\left[\left(\frac{\pi}{2}-\phi_{s}\right) \sin \phi_{s}-\cos \phi_{s}\right]\right|}$, there are two series of pendulumlike rf buckets with their stable fixed points separated by $\left|\eta_{0} / \eta_{1}\right|$. Middle: The two series merge when $\left|\eta_{0} / \eta_{1}\right|$ is reduced to the critical value. Bottom: Further reduction of $\left|\eta_{0} / \eta_{1}\right|$ to below the critical value causes the stable and unstable fixed points to pair differently, top and bottom instead of side by side. The pendulum-shape buckets change drastically to $\alpha$-shape. All the stable fixed points are denoted by small circles. The unstable fixed points are where the separatrices cross each other. 
Consider next the fixed point $\left(\delta_{f}, \Delta \phi_{f}\right)=\left(\eta_{0} /\left|\eta_{1}\right|, 2 n \pi\right)$ Letting $\delta=\delta_{f}+\Delta \delta$, one gets

$$
\begin{aligned}
& \frac{d \Delta \phi}{d t}=-h \omega_{0 s} \eta_{0} \Delta \delta, \\
& \frac{d \Delta \delta}{d t}=\frac{e V \cos \phi_{s}}{E_{s} T_{s}} \Delta \phi,
\end{aligned}
$$

implying that the fixed point is unstable when $\eta \cos \phi_{s}<0$. On the other hand, near the fixed point $\left(\delta_{f}, \Delta \phi_{f}\right)=\left(\eta_{0} /\left|\eta_{1}\right|,(2 n+1) \pi\right)$, the equations of motion are

$$
\begin{aligned}
& \frac{d \Delta \phi}{d t}=-h \omega_{0 s} \eta_{0} \Delta \delta \\
& \frac{d \Delta \delta}{d t}=-\frac{e V \cos \phi_{s}}{E_{s} T_{s}} \Delta \phi,
\end{aligned}
$$

showing that this fixed point is stable. We see that the small-amplitude synchrotron tune is again given by Eq. (2.9), and is exactly the same as around the other stable fixed point.

\section{Separatrices}

The Hamiltonian from which the equations of motion are derived can be written as

$$
H=h \omega_{0 s}\left(\frac{\eta_{0} \delta^{2}}{2}-\frac{\left|\eta_{1}\right| \delta^{3}}{3}\right)+\frac{e V \omega_{0 s}}{2 \pi E_{s}}\left[\cos \left(\Delta \phi+\phi_{s}\right)+\Delta \phi \sin \phi_{s}\right]
$$

The separatrix that passes through the unstable fixed point $\left(0,(2 n+1) \pi-2 \phi_{s}\right)$ is

$$
\left(\frac{\eta_{0} \delta^{2}}{2}-\frac{\left|\eta_{1}\right| \delta^{3}}{3}\right)+\frac{e V}{2 \pi E_{s} h}\left\{\cos \left(\Delta \phi+\phi_{s}\right)+\Delta \phi \sin \phi_{s}\right\}=\frac{e V}{2 \pi E_{s} h}\left\{-\cos \phi_{s}+\left[(2 n+1) \pi-2 \phi_{s}\right] \sin \phi_{s}\right\}
$$

or

$$
\left(\frac{\eta_{0} \delta^{2}}{2}-\frac{\left|\eta_{1}\right| \delta^{3}}{3}\right)=\frac{e V}{2 \pi E_{s} h}\left\{\left[(2 n+1) \pi-\Delta \phi-2 \phi_{s}\right] \sin \phi_{s}-\cos \left(\Delta \phi+\phi_{s}\right)-\cos \phi_{s}\right\},
$$

There is also another branch of the separatrix that passes through the fixed point $\left(\eta_{0} /\left|\eta_{1}\right|, 2 n \pi\right)$. The moment when these two branches merge is obtained by substituting $(\delta, \Delta \phi)=\left(\eta_{0} /\left|\eta_{1}\right|, 2 n \pi\right)$ into Eq. (2.14) and is represented by

$$
\left(\frac{\eta_{0}}{\left|\eta_{1}\right|}\right)_{c}=\left\{\frac{6 e V}{\pi E_{s} h \eta_{0}}\left[\left(\frac{\pi}{2}-\phi_{s}\right) \sin \phi_{s}-\cos \phi_{s}\right]\right\}^{1 / 2}
$$

When $\eta_{0} /\left|\eta_{1}\right|<\left(\eta_{0} /\left|\eta_{1}\right|\right)_{c}$, we have the ordinary pendulum-like bucket, i.e., with $(0,(2 n+$ 1) $\left.\pi-2 \phi_{s}\right)$ as the unstable fixed point for the bucket centering at the stable fixed point 
$\left(0,\left(2 n \pi+\phi_{s}\right)\right)$, while $\left(\eta_{0} /\left|\eta_{1}\right|, 2 n \pi+\phi_{s}\right)$ as the unstable fixed point for the bucket centering at the stable fixed point $\left(\eta_{0} /\left|\eta_{1}\right|,(2 n+1) \pi-2 \phi_{s}\right)$. At $\eta_{0} /\left|\eta_{1}\right|=\left(\eta_{0} /\left|\eta_{1}\right|\right)_{c}$, these two series of buckets merge. When $\eta_{0} /\left|\eta_{1}\right|>\left(\eta_{0} /\left|\eta_{1}\right|\right)_{c}$, the buckets become tear drops or $\alpha$-like, with $\left(\eta_{0} /\left|\eta_{1}\right|, 2 n \pi+\phi_{s}\right)$ as the unstable fixed point for the bucket centering at the stable fixed point $\left(0,\left(2 n \pi+\phi_{s}\right)\right)$, and $\left(0,(2 n+1) \pi-2 \phi_{s}\right)$ as the unstable fixed point for the bucket centering at the stable fixed point $\left(\eta_{0} /\left|\eta_{1}\right|,(2 n+1) \pi-2 \phi_{s}\right)$.

One important remark is that although the branches of the separatrix move and merge, $\left(0,\left(2 n \pi+\phi_{s}\right)\right)$ and $\left(\eta_{0} /\left|\eta_{1}\right|,(2 n+1) \pi-2 \phi_{s}\right)$ remain stable fixed points. This implies that the small-amplitude oscillations about the stable fixed point would not experience sudden changes during the merging of the separatrix branches.

\section{Plotting the Separatrices}

The separatrix in Eq. (2.14) can be plotted by setting a value of $\Delta \phi$ and solving a cubic equation to obtain $\delta$. First let us perform the normalization

$$
\begin{aligned}
& \bar{\eta}_{0}=\frac{2 \pi E_{s} h}{e V} \eta_{0}, \\
& \bar{\eta}_{1}=\frac{2 \pi E_{s} h}{e V} \eta_{1} .
\end{aligned}
$$

We then have

$$
\frac{\bar{\eta}_{0} \delta^{2}}{2}-\frac{\bar{\eta}_{1} \delta^{3}}{3}=a
$$

where

$$
a=\left\{\left[(2 n+1) \pi-\Delta \phi-2 \phi_{s}\right] \sin \phi_{s}-\cos \left(\Delta \phi+\phi_{s}\right)-\cos \phi_{s}\right\} .
$$

We next cast the equation into the form

$$
\delta^{-3}-\frac{\bar{\eta}_{0}}{2 a} \delta^{-1}=-\frac{\left|\bar{\eta}_{1}\right|}{3 a} .
$$

Now substitute for $\delta^{-1}=b x$ to obtain

$$
x^{3}-\frac{\bar{\eta}_{0}}{2 a b^{2}} x=-\frac{\left|\bar{\eta}_{1}\right|}{3 a b^{3}} .
$$

If $a>0$, identify

$$
b=-\sqrt{\frac{2 \bar{\eta}_{0}}{3 a}} .
$$


Let

$$
c=-\frac{4\left|\bar{\eta}_{1}\right|}{3 a b^{3}}>0
$$

If $c<1$, we get

$$
\cos 3 A=c \quad \text { so that } A=\frac{1}{3}\left[\cos ^{-1}\left|\frac{4 \bar{\eta}_{1}}{3 a b^{3}}\right|+2 \pi n\right], \quad n=0,1,2 .
$$

Then the three solutions are given by $\delta=(b \cos A)^{-1}$. If $c>1$, we get the solution

$$
\cosh 3 A=c \quad \text { so that } A=\frac{1}{3} \cosh ^{-1}\left(\frac{4 \bar{\eta}_{1}}{3 a\left|b^{3}\right|}\right) .
$$

In this case, there is only one real solution $\delta=(b \cosh A)^{-1}$.

When $a<0$, we have instead

$$
x^{3}+\frac{\bar{\eta}_{0}}{2|a| b^{2}} x=\frac{\left|\bar{\eta}_{1}\right|}{3|a| b^{3}} .
$$

We can identify

$$
b=\sqrt{\frac{2 \bar{\eta}_{0}}{3|a|}}
$$

We obtain

$$
\sinh 3 A=c \text { so that } A=\frac{1}{3} \sinh ^{-1}\left(\frac{4 \bar{\eta}_{1}}{3|a| b^{3}}\right),
$$

and there is only one real solution $\delta=(b \sinh A)^{-1}$.

To plot the separatrices, let us assign some reasonable value to $\bar{\eta}_{0}$. One example is with $E_{s}=1 \mathrm{GeV}, h=200, V=100 \mathrm{kV}$, and $\eta_{0}=0.001$, arriving at $\bar{\eta}_{0}=1.26 \times 10^{10}$. For simplicity, let us assign just $\bar{\eta}_{0}=1 \times 10^{6}$. The synchronous phase is chosen to be $\phi_{s}=0.9 \pi$. Then the critical $\eta_{1}$ turns out to be $\left|\eta_{1}\right| / \eta_{0}=38.482$. The first example is for $\left|\eta_{1}\right| / \eta_{0}=28$. We show in Fig. 2 the two separatrix branches with $n=0$. We do see the upper (red) and lower (black) buckets resemble the fish-like accelerating buckets, but mingled together. What is not expected are two small isolated circles. We next decrease $\left|\eta_{1}\right| / \eta_{0}$, the small isolated circles become larger and the two branches of the separatrix bulging out towards the circles. When $\left|\eta_{1}\right| / \eta_{0}=23.315$, each isolated circle becomes the separatrix of adjacent branch, as shown in the second-row left figure. We can say that the $n=0$ upper branch coincides exactly with the $n=1$ lower branch, while the $n=0$ lower branch coincides exactly with the $n=-1$ upper branch. Now instead of one, each branch has two unstable fixed points and two stable fixed points. When $\left|\eta_{1}\right| / \eta_{0}$ is further decreased, the $n=0$ lower branch 

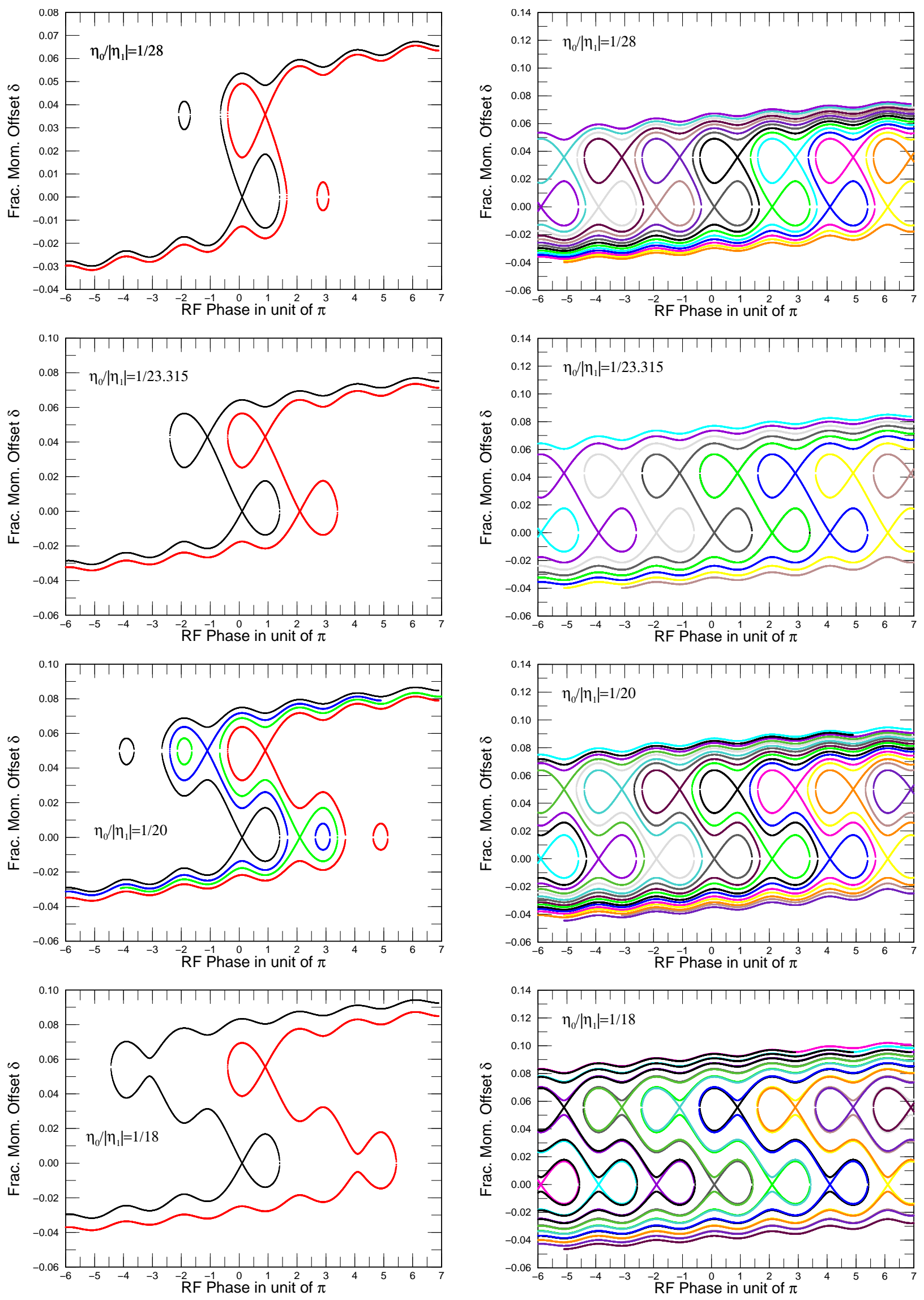

Figure 2: Plot of the $n=0$ upper and lower separatrix branches on the left and more adjacent branches on the right, with $\phi_{s}=0.9 \pi \cdot \eta_{0} /\left|\eta_{1}\right|$ increases gradually from row to row. The last row is on the next page. 

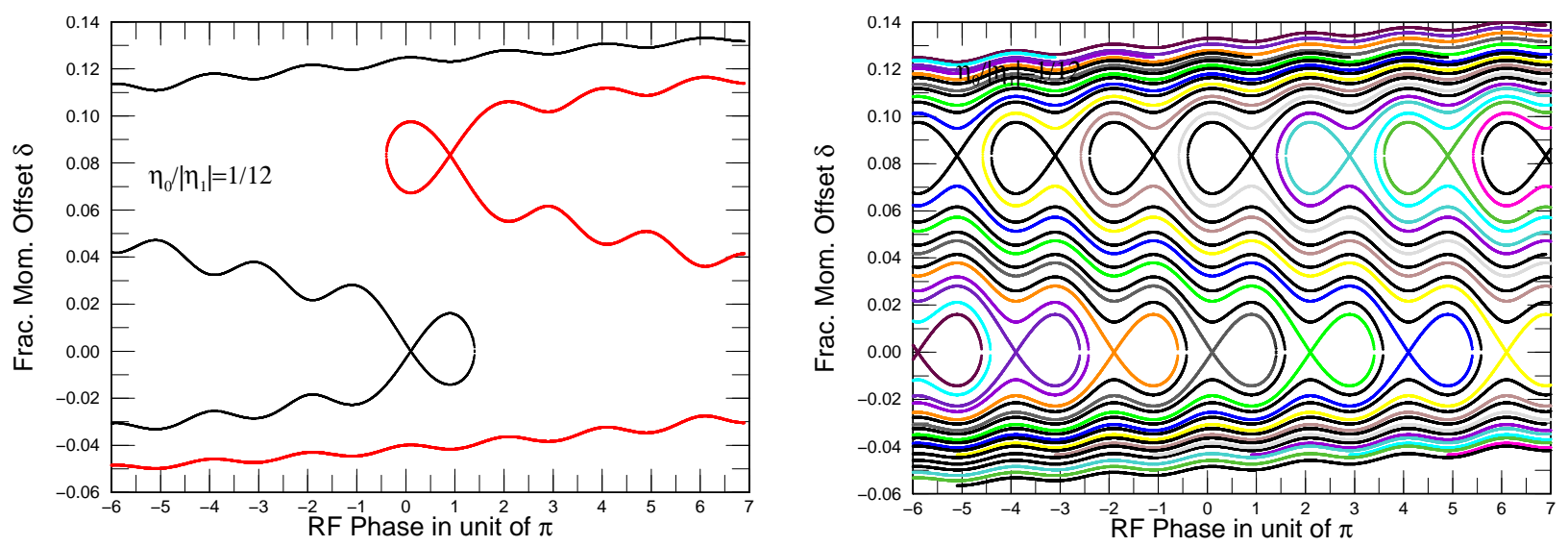

(black) has the new unstable fixed point opened up and wrapped around the $n=-1$ upper branch (blue). At the same time, a new isolated circle appears to the left. In this plot, we shown not only the $n=0$ upper and lower separatrix branches (black and red), but also the $n=-1$ upper branch (blue) and the $n=1$ lower branch (green). We see in the following rows that, as $\left|\eta_{1}\right| / \eta_{0}$ decreases further, the $n=0$ lower branch (black) gradually spread out to the right near the upper series buckets $\left(\delta=\eta_{0} /\left|\eta_{1}\right|\right)$. Finally as $\eta_{0} /\left|\eta_{1}\right|$ becomes very very large, the upper and lower series of buckets appear to be separated and look exactly like those buckets with $\eta_{1}=0$.

From the above study, it is clear that the small isolated circles are not part of the separatrix. They are just elliptical trajectories about the next stable fixed point and happen to have exactly the same Hamiltonian value as the $n=0$ separatrix branches under study. For this reason, these isolated circles are removed, and we plot all adjacent branches of the separatrix on the right of the rows. We see that all those plots are very busy. In general, there are $h$ upper branches and $h$ lower branches where $h$ is the rf harmonic. At the upper and lower edges, there are $h$ wavy separatrices clustered together, exactly in the same way as in the situation when $\eta_{1}=0$.

When $\eta_{0} /\left|\eta_{1}\right|$ reaches the critical value given in Eq. 2.15, the upper and lower series of buckets merge, as depicted in the upper plots of Fig. 3. Further reduction of $\eta_{0} /\left|\eta_{1}\right|$ produces the $\alpha$-like or tear-drop buckets, as depicted in the lower plots.

When the synchronous phase $\phi_{s}=0$ or $\pi$, the separatrices are very much simpler, because all the individual accelerating buckets corresponding to the same momentum series collapse into one long string of connected buckets. They are depicted in Fig. 1 and also Fig. 4 when $\eta_{0} /\left|\eta_{1}\right|$ is larger, equal, or equal to the critical value determined by Eq. (2.15). 

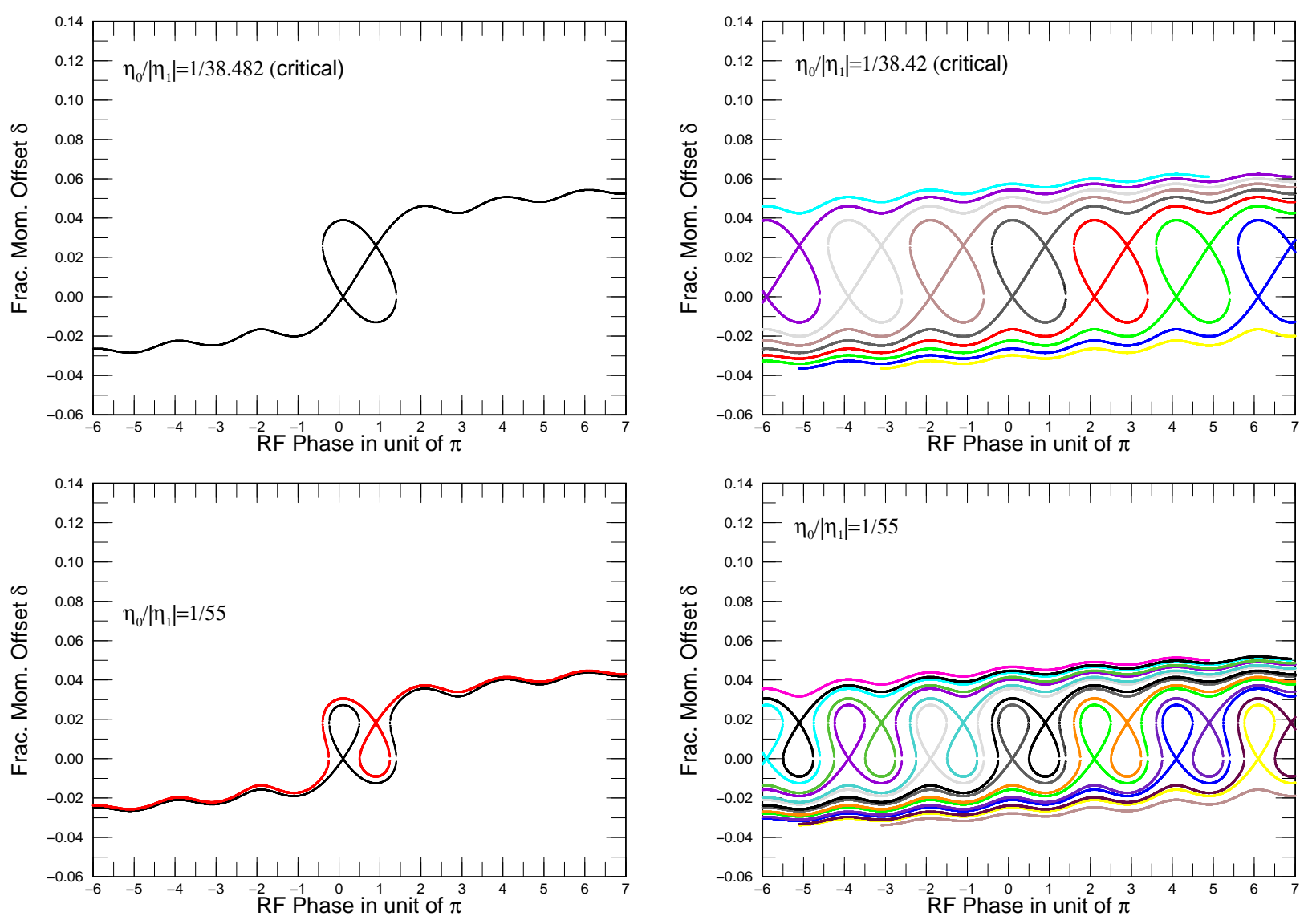

Figure 3: Plot of the $n=0$ upper and lower separatrix branches on the left and more adjacent branches on the right. First row is when $\eta_{0} /\left|\eta_{1}\right|=1 / 38.482$, its critical value. The upper and lower branches merge. When $\eta_{0} /\left|\eta_{1}\right|$ is further increased, the two branches split leaving the buckets in another shape, with stable and unstable fixed points at the same $\operatorname{rf}$ phase, but $\delta=0$, and $\eta_{0} /\left|\eta_{1}\right|$. On the other hand, before the merging when $\eta_{0} /\left|\eta_{1}\right|>1 / 38.482$, each bucket has its stable and unstable points at the same $\delta$ (either 0 or $\left.\eta_{0} /\left|\eta_{1}\right|\right)$ but different rf phase. 

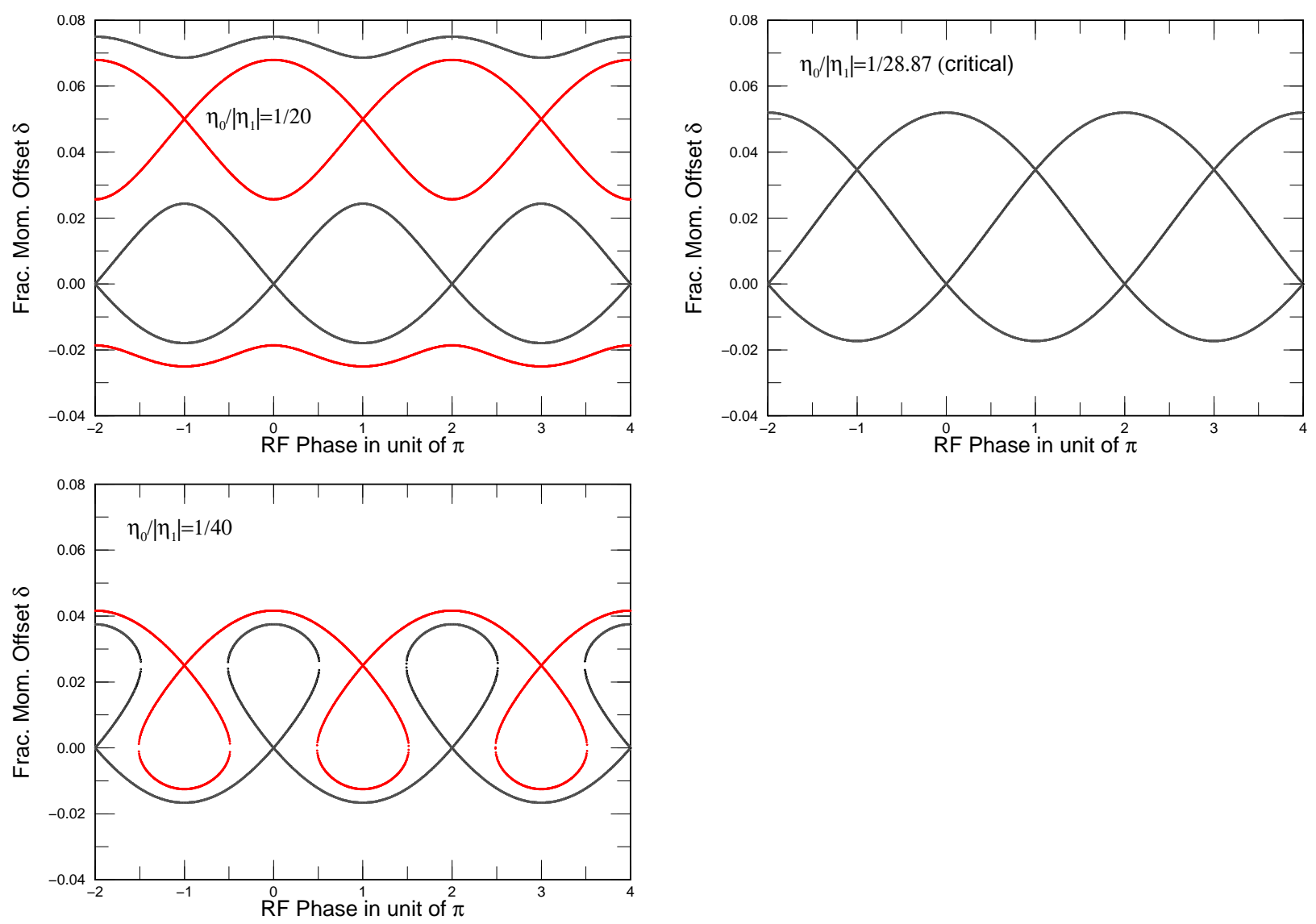

Figure 4: The plots are the same as before, but when $\phi_{s}=\pi$. All the plots are much more simplied. The first, second, and third plots are when $\eta_{0} /\left|\eta_{1}\right|$ larger than, equal to, and smaller than the critical value.

\section{Measurement of $\eta_{0}$ and $\eta_{1}$}

The first two or three expansion coefficients of the slip factor can be measured by varying the rf frequency. This push the closed orbit inward or outward. The energy or momentum of the particles synchronized to the new rf frequency is now changed and so will be the synchrotron tune. By measuring the synchrotron tune as a function of rf frequency, $\eta_{0}$ and $\eta_{1}$ can be inferred. To accomplish this, we must derive the equations of motion at this new synchronous momentum $p_{1}$. 


\section{Phase Equation}

The rate of change in time advance is

$$
\frac{d \Delta \tau}{d t}=\frac{\tau-\tau_{1}}{T_{1}}
$$

where $T_{1}=2 \pi / \omega_{1}$ is the revolution frequency of the particles synchronized to the new $\mathrm{rf}$ frequency $\omega_{\mathrm{rf}}=h \omega_{1}$, and $\tau_{1}$ is the time advance of the new synchronous particles relative to the old one. Note that we are still using the old synchronous particles as a reference. In other words, in above $\tau$ is the arrival time of a test particle in advance of the old synchronous particles. We wish to point out that we are just using the old synchronous particles as a mathematical reference. We do this because we have already the equations of motion and all expansion referenced to the old synchronous particles. We then have

$$
\frac{d \Delta \tau}{d t}=\frac{T_{0}}{T_{1}}\left(\frac{\tau-\tau_{0}}{T_{0}}-\frac{\tau_{1}-\tau_{0}}{T_{0}}\right) .
$$

Notice that we are changing our notations. The subscript ' 0 ' denotes quantities synchronized to the old rf frequency, instead of the subscript 's' used in the first section.

In Eq. (4.29) obviously $\tau_{0}=0$, because the old synchronous particle is always on time. With $\delta=\left(p-p_{0}\right) / p_{0}$ and $\delta_{1}=\left(p_{1}-p_{0}\right) / p_{0}$, Eq. (4.29) becomes

$$
\begin{aligned}
\frac{d \Delta \tau}{d t} & =-\frac{T_{0}}{T_{1}}\left[\left(\eta_{0} \delta+\eta_{1} \delta^{2}+\eta_{2} \delta^{3}+\cdots\right)-\left(\eta_{0} \delta_{1}+\eta_{1} \delta_{1}^{2}+\eta_{2} \delta_{1}^{3}+\cdots\right)\right] \\
& =-\frac{T_{0}}{T_{1}}\left[\left(\eta_{0}+2 \eta_{1} \delta_{1}+3 \eta_{2} \delta_{1}^{2}+\cdots\right) \Delta+\frac{1}{2} \Delta^{2}\left(2 \eta_{1}+6 \eta_{2} \delta_{1}+\cdots\right)+\mathcal{O}\left(\Delta^{3}\right)\right]
\end{aligned}
$$

where $\Delta=\delta-\delta_{1}=\left(p-p_{1}\right) / p_{0}$. The rf phase advance is still given by $\Delta \phi=-h \omega_{1} \tau$. We therefore have the phase equation

$$
\frac{d \Delta \phi}{d t}=-h \omega_{1} \frac{d \Delta \tau}{d t}=\frac{h \omega_{1} T_{0}}{T_{1}}\left[\left(\eta_{0}+2 \eta_{1} \delta_{1}+3 \eta_{2} \delta_{1}^{2}+\cdots\right) \Delta+\mathcal{O}\left(\Delta^{2}\right)\right] .
$$

\section{Energy Equation}

Since the momentum offset $\Delta$ has been used as a canonical variable, the energy $E$ in the energy equation must be converted to momentum also. In other words, we are now including the fact the the particles are not ultra-relativistic. Here, $E=\sqrt{p^{2}+m^{2}}$ with $m$ the particle 
mass and the velocity of light put to unity. Let us expand around the old synchronous particle momentum $p_{0}$. We have

$$
\frac{d E}{d p}=\frac{p}{\sqrt{p^{2}+m^{2}}}=\frac{p}{E}, \quad \frac{d^{2} E}{d p^{2}}=\frac{m^{2}}{\left(p^{2}+m^{2}\right)^{3 / 2}}=\frac{m^{2}}{E^{3}}, \quad \frac{d^{2} E}{d p^{2}}=-\frac{3 m^{2} p}{\left(p^{2}+m^{2}\right)^{5 / 2}}=\frac{3 m^{2} p}{E^{5}},
$$

and therefore

$$
\frac{\Delta E}{E_{0}}=\frac{E-E_{0}}{E_{0}}=\beta_{0}^{2} \delta+\frac{\beta_{0}^{2}}{2 \gamma_{0}^{2}} \delta^{2}-\frac{\beta_{0}^{4}}{2 \gamma_{0}^{2}} \delta^{3}+\mathcal{O}\left(\delta^{4}\right)
$$

Then

$$
\begin{aligned}
\frac{d \delta}{d t} & =\frac{1}{\beta_{0}^{2}} \frac{d}{d t}\left(\frac{\Delta E}{E_{0}}\right)\left(1+\frac{\delta}{\gamma^{2}}-\frac{3 \beta_{0}^{2}}{2 \gamma_{0}^{2}} \delta^{2}+\cdots\right)^{-1} \\
& =\frac{e V}{\beta_{0}^{2} E_{0} T_{0}}\left[\sin \left(\Delta \phi+\phi_{s}\right)-\sin \phi_{s}\right]\left(1-\frac{\delta}{\gamma^{2}}+\frac{3 \gamma_{0}^{2} \beta_{0}^{2}+2}{2 \gamma_{0}^{4}} \delta^{2}+\cdots\right) .
\end{aligned}
$$

This is the energy equation for a particle in the old reference rf, when the particle is not ultra relativistic. The small-amplitude synchrotron tune requires only the linear part of the equation. Thus the synchrotron tune $\nu_{0}$ is given by

$$
\nu_{s 0}^{2}=\frac{h e V \cos \phi_{s} \eta_{0}}{2 \pi \beta^{2} E_{0}}
$$

where the expansion of $E$ in terms of $p$ does not contribute at all.

We now derive the energy equation at the new rf frequency $h \omega_{1}$. We have

$$
\begin{aligned}
\frac{\Delta E_{1}}{E_{0}} & =\frac{E-E_{1}}{E_{0}}=\frac{\left(E-E_{0}\right)-\left(E_{1}-E_{0}\right)}{E_{0}} \\
& =\left(\beta_{0}^{2} \delta+\frac{\beta_{0}^{2}}{2 \gamma_{0}^{2}} \delta^{2}-\frac{\beta_{0}^{4}}{2 \gamma_{0}^{2}} \delta^{3}+\cdots\right)-\left(\beta_{0}^{2} \delta_{1}+\frac{\beta_{0}^{2}}{2 \gamma_{0}^{2}} \delta_{1}^{2}-\frac{\beta_{0}^{4}}{2 \gamma_{0}^{2}} \delta_{1}^{3}+\cdots\right) \\
& =\left(\beta_{0}^{2}+\frac{\beta_{0}^{2}}{\gamma_{0}^{2}} \delta_{1}-\frac{3 \beta_{0}^{4}}{2 \gamma_{0}^{2}} \delta_{1}^{2}+\cdots\right) \Delta+\frac{\Delta^{2}}{2}\left(\frac{\beta_{0}^{2}}{\gamma_{0}^{2}}-\frac{3 \beta_{0}^{4}}{\gamma_{0}^{2}} \delta_{1}+\cdots\right)+\mathcal{O}\left(\Delta^{3}\right),
\end{aligned}
$$

with $\Delta=\delta-\delta_{1}$. Notice the notation that $\Delta E_{1}=E-E_{1}$ and is not $\Delta E=E_{1}-E_{0}$. We then have

$$
\frac{d}{d t}\left(\frac{\Delta E_{1}}{E_{0}}\right)=\left[\left(\beta_{0}^{2}+\frac{\beta_{0}^{2}}{\gamma_{0}^{2}} \delta_{1}-\frac{3 \beta_{0}^{4}}{2 \gamma_{0}^{2}} \delta_{1}^{2}+\cdots\right)+\Delta\left(\frac{\beta_{0}^{2}}{\gamma_{0}^{2}}-\frac{3 \beta_{0}^{4}}{\gamma_{0}^{2}} \delta_{1}+\cdots\right)+\mathcal{O}\left(\Delta^{2}\right)\right] \frac{d \Delta}{d t} .
$$


We can write

$$
\begin{aligned}
\frac{d \Delta}{d t} & \approx \frac{1}{\beta_{0}^{2}} \frac{d}{d t}\left(\frac{\Delta E}{E_{0}}\right)\left[\left(1+\frac{\delta_{1}}{\gamma_{0}^{2}}-\frac{3 \beta_{0}^{2}}{2 \gamma_{0}^{2}} \delta_{1}^{2}\right)+\frac{\Delta}{\gamma_{0}^{2}}\left(1-3 \beta_{0}^{2} \delta_{1}\right)\right]^{-1} \\
& \approx \frac{1}{\beta_{0}^{2}} \frac{d}{d t}\left(\frac{\Delta E}{E_{0}}\right)\left[\left(1-\frac{\delta_{1}}{\gamma_{0}^{2}}+\frac{3 \beta_{0}^{2}}{2 \gamma_{0}^{2}} \delta_{1}^{2}\right)-\frac{\Delta}{\gamma_{0}^{2}}\left(1-3 \beta_{0}^{2} \delta_{1}\right)+\left(\frac{\delta_{1}}{\gamma_{0}^{2}}+\frac{\Delta}{\gamma_{0}^{2}}\right)^{2}\right] \\
& \approx \frac{1}{\beta_{0}^{2}} \frac{d}{d t}\left(\frac{\Delta E}{E_{0}}\right)\left[\left(1-\frac{\delta_{1}}{\gamma_{0}^{2}}+\frac{3 \gamma_{0}^{2} \beta_{0}^{2}+2}{2 \gamma_{0}^{4}} \delta_{1}^{2}\right)-\frac{\Delta}{\gamma_{0}^{2}}\left(1-\frac{3 \gamma_{0}^{2} \beta_{0}^{2}+2}{\gamma_{0}^{2}} \delta_{1}\right)\right] .
\end{aligned}
$$

We finally arrive at

$$
\frac{d \Delta}{d t} \approx \frac{e V}{\beta_{0}^{2} E_{0} T_{1}}\left[\sin \left(\Delta \phi+\phi_{s}\right)-\sin \phi_{s}\right]\left[\left(1-\frac{\delta_{1}}{\gamma_{0}^{2}}+\frac{3 \gamma_{0}^{2} \beta_{0}^{2}+2}{2 \gamma_{0}^{4}} \delta_{1}^{2}\right)-\frac{\Delta}{\gamma_{0}^{2}}\left(1-\frac{3 \gamma_{0}^{2} \beta_{0}^{2}+2}{\gamma_{0}^{2}} \delta_{1}\right)\right]
$$

Thus the small-amplitude synchrotron tune $\nu_{s 1}$ is given by

$$
\begin{aligned}
\nu_{s 1}^{2} & \approx-\frac{h \eta_{0} e V \cos \phi_{s}}{2 \pi \beta^{2} E_{0}} \frac{T_{0}}{T_{1}}\left(1+\frac{2 \eta_{1}}{\eta_{0}} \delta_{1}+\frac{3 \eta_{2}}{\eta_{0}} \delta_{1}^{2}\right)\left(1-\frac{\delta_{1}}{\gamma_{0}^{2}}+\frac{3 \gamma_{0}^{2} \beta_{0}^{2}+2}{2 \gamma_{0}^{4}} \delta_{1}^{2}\right) \\
& =-\frac{h \eta_{0} e V \cos \phi_{s}}{2 \pi \beta^{2} E_{0}}\left[1-\eta_{0} \delta_{1}+\left(\eta_{0}^{2}-\eta_{1}\right) \delta_{1}^{2}\right]\left[1+\left(\frac{2 \eta_{1}}{\eta_{0}}-\frac{1}{\gamma_{0}^{2}}\right) \delta_{1}+\left(\frac{3 \eta_{2}}{\eta_{0}}+\frac{3 \gamma_{0}^{2} \beta_{0}^{2}+2}{2 \gamma_{0}^{4}}-\frac{2 \eta_{1}}{\eta_{0} \gamma_{0}^{2}}\right) \delta_{1}^{2}\right] \\
& =-\frac{h \eta_{0} e V \cos \phi_{s}}{2 \pi \beta^{2} E_{0}}\left[1+\left(\frac{2 \eta_{1}}{\eta_{0}}-\frac{1}{\gamma_{0}^{2}}-\eta_{0}\right) \delta_{1}+\left(\frac{3 \eta_{2}}{\eta_{0}}+\frac{3 \gamma_{0}^{2} \beta_{0}^{2}+2}{2 \gamma_{0}^{4}}+\frac{\left(\eta_{0}^{2}-2 \eta_{1}\right)}{\eta_{0} \gamma_{0}^{2}}+\eta_{0}^{2}-3 \eta_{1}\right) \delta_{1}^{2}\right] .
\end{aligned}
$$

The change in rf frequency is related to the change in momentum offset according to

$$
\frac{\Delta \omega_{\mathrm{rf}}}{\omega_{\mathrm{rf}}^{0}}=\frac{\omega_{1}-\omega_{0}}{\omega_{0}}=\frac{T_{0}-T_{1}}{T_{1}}=-\frac{\Delta T_{1}}{T_{0}} \frac{1}{\Delta T_{1} / T_{0}+1} \approx-\eta_{0} \delta_{1}+\left(\eta_{0}^{2}-\eta_{1}\right) \delta_{1}^{2} .
$$

Inverse solving yields

$$
\delta_{1}=-\frac{1}{\eta_{0}}\left(\frac{\Delta \omega_{\mathrm{rf}}}{\omega_{\mathrm{rf}}^{0}}\right)+\frac{\eta_{0}^{2}-\eta_{1}}{\eta_{0}^{3}}\left(\frac{\Delta \omega_{\mathrm{rf}}}{\omega_{\mathrm{rf}}^{0}}\right)^{2} .
$$

Substituting into Eq. (4.40) gives

$$
\nu_{s 1}^{2} \approx-\frac{h \eta_{0} e V \cos \phi_{s}}{2 \pi \beta^{2} E_{0}}\left[1+\frac{s_{1}}{\eta_{0}}\left(\frac{\Delta \omega_{\mathrm{rf}}}{\omega_{\mathrm{rf}}^{0}}\right)+\frac{s_{2}}{\eta_{0}^{2}}\left(\frac{\Delta \omega_{\mathrm{rf}}}{\omega_{\mathrm{rf}}^{0}}\right)^{2}\right],
$$

with the coefficients

$$
\begin{aligned}
& s_{1}=-\frac{2 \eta_{1}-\eta_{0}^{2}}{\eta_{0}}+\frac{1}{\gamma_{0}^{2}}, \\
& s_{2}=\frac{3 \eta_{2} \eta_{0}-2 \eta_{1}^{2}}{\eta_{0}^{2}}-\frac{\eta_{1}}{\eta_{0} \gamma_{0}^{2}}+\frac{3 \gamma_{0}^{2} \beta_{0}^{2}+2}{2 \gamma_{0}^{4}} .
\end{aligned}
$$


In practice, the fractional shift in rf frequency is usually of the order $\eta_{0}$, or $\Delta \omega_{\text {rf }} / \omega_{\text {rf }}^{0} \sim \eta_{0}$, which is small near transition. Thus this experiment must be performed with high accuracy.

A formula similar to Eq. (4.44) is given by Nadji, et. al. [1] and derived in a different way for ultra-relativistic beam particles, ${ }^{*}$

$$
\nu_{s 1}^{2} \approx-\frac{h \alpha_{0} e V \cos \phi_{s}}{2 \pi E_{0}}\left[1-\frac{4 \alpha_{1}}{\alpha_{0}^{2}}\left(\frac{\Delta \omega_{\mathrm{rf}}}{\omega_{\mathrm{rf}}^{0}}\right)\right]^{1 / 2},
$$

where we define the momentum-compaction expansion as

$$
\frac{\Delta C}{C_{0}}=\alpha_{0} \delta+\alpha_{1} \delta^{2}+\alpha_{2} \delta^{3}+\cdots,
$$

with $C_{0}$ being the length of the closed orbit synchronous at the unperturbed rf frequency and $\Delta C$ being the change in closed-orbit length at momentum offset $\delta$. As $\beta \rightarrow 1$, one should make the substitutions $\eta_{0} \rightarrow \alpha_{0}, \eta_{1} \rightarrow \alpha_{2}, \eta_{2} \rightarrow \alpha_{2}$, etc. Nadji's formula of Eq. (4.45) agrees with our Eq. (4.44) up to the first order in $\Delta \omega_{\mathrm{rf}} / \omega_{\mathrm{rf}}^{0}$. It also agrees in the next order provided that $\alpha_{2}=0$.

\section{References}

[1] A. Nadji, P. Brunelle, G. Flynn, M.P. Level, M.Sommer, H. Zyngier, Quasi-isochronous experiments with the Super-ACO stroage ring, Nucl. Inst. Meth. A378, 376 (1996).

\footnotetext{
${ }^{*}$ In Nadji's paper, their $\alpha_{i}$ 's are our $\alpha_{i-1}$ 's.
} 